\title{
Supporting Information: Triplet Excitons in Pentacene are Intrinsically Difficult to Dissociate via Charge Transfer
}

Natalie A. Pace, ${ }^{1,2}$ Tyler T. Clikeman, ${ }^{3}$ Steven H. Strauss, ${ }^{3}$ Olga V. Boltalina, ${ }^{3}$ Justin C.

$$
\text { Johnson, },^{*} \text { Garry Rumbles, }{ }^{1,2,4^{*}} \text { and Obadiah G. Reid }{ }^{1,4^{*}}
$$

${ }^{1}$ National Renewable Energy Laboratory, 15013 Denver West Parkway, Golden, Colorado 80401, USA

${ }^{2}$ Department of Chemistry, University of Colorado Boulder, Boulder, Colorado 80309, USA

${ }^{3}$ Department of Chemistry, Colorado State University, Fort Collins, Colorado 80523, USA

${ }^{4}$ Renewable and Sustainable Energy Institute, University of Colorado Boulder, Boulder, Colorado 80309, USA

*Corresponding author email address: Obadiah.Reid@colorado.edu

*Corresponding author email address: Justin.Johnson@nrel.gov

*Corresponding author email address: Garry.Rumbles@nrel.gov 
This supplementary information contains the following sections:

1. Absorbance Spectrum of Each Component

2. Surface to Volume Ratio of a Spherical Particle

3. Full TA Image Fits, Residuals, and Kinetic Schemes

4. Kinetic Monte Carlo Modeling of Diffusion-Limited Effective Charge Transfer Rate Constants 


\section{Absorbance Spectrum of Each Component}

As shown in the absorption spectra (Figure S1), the lowest-energy excited singlet states of the three components have the following energetic ordering: $\mathrm{S}_{1}(\mathrm{PbPc})<\mathrm{S}_{1}$ (pentacene) $<$ $\mathrm{S}_{1}(\mathrm{PDI})$. As a result, singlet energy transfer cannot occur from $\mathrm{PbPc}$ to pentacene or PDI molecules or from pentacene to PDI molecules.

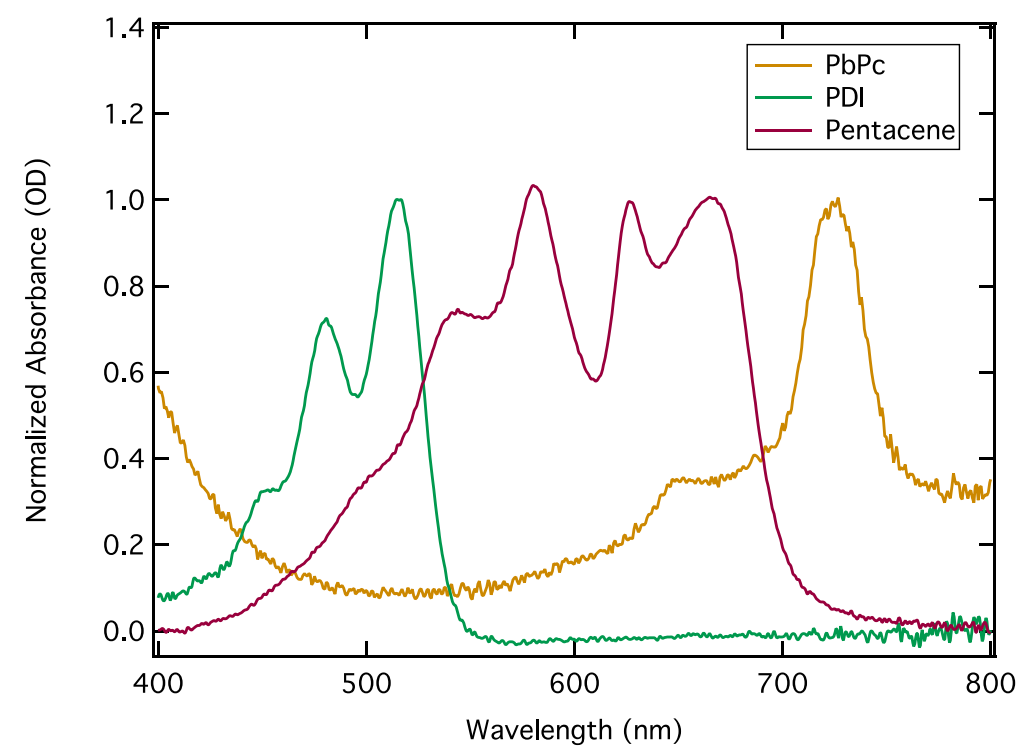

S1: Absorbance spectra for neat pentacene, polystyrene $10.01 \mathrm{~m} \mathrm{PbPc}$, and polystyrene $/ 0.01 \mathrm{~m}$ $\mathrm{PDI}(\mathrm{F} 7 \mathrm{Bu})-\left(\mathrm{CF}_{3}\right)_{3}$. 


\section{Surface to Volume Site Ratio of a Spherical Particle}

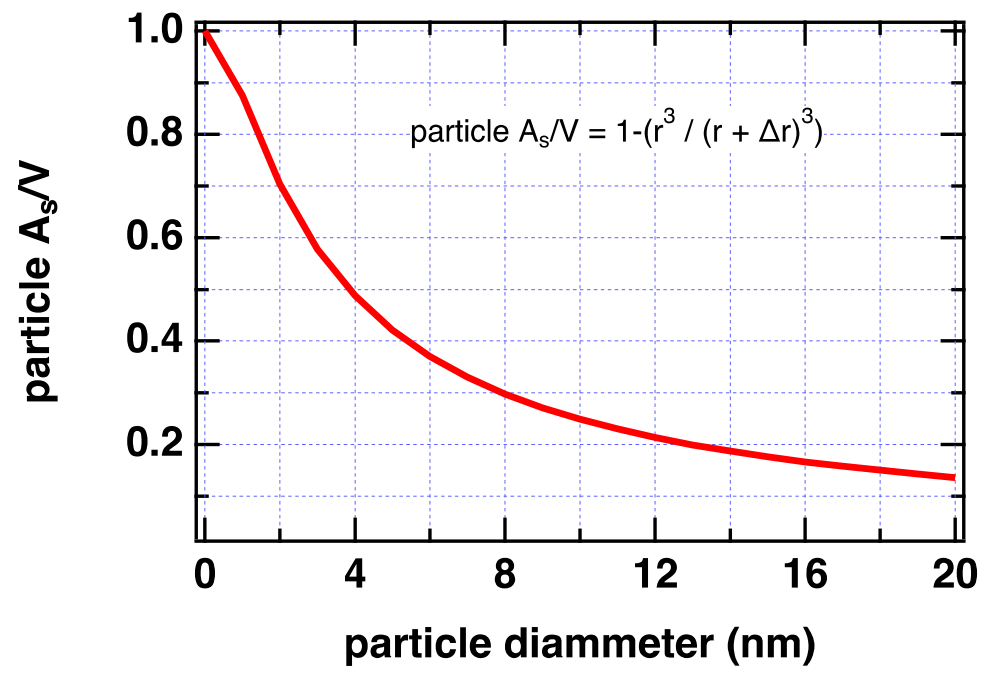

S2) Surface to volume site ratio as a function of particle diameter, assuming a spherical particle with cubic sites, $0.5 \mathrm{~nm}$ on a side $(\Delta r=0.5 \mathrm{~nm})$.

For a spherical particle with radius $r+\Delta r$, that contains a discrete number of molecular sites with volume $\Delta \mathrm{r}^{3}$, the total number of sites is given by:

$$
N=\frac{4}{3} \pi(r+\Delta r)^{3} / \Delta r^{3}
$$

And the number of sites on the surface is given by:

$$
N_{s}=\frac{\frac{4}{3} \pi(r+\Delta r)^{3}}{\Delta r^{3}}-\frac{4}{3} \pi r^{3} / \Delta r^{3}
$$

The surface to volume site ratio is then $\mathrm{N}_{\mathrm{s}} / \mathrm{N}$ :

$$
R_{S V}=1-r^{3} /(r+\Delta r)^{3}
$$




\section{Full TA Data, Fits, and Fitting Details:}

\section{Fitting Details}

In this section we provide details of our kinetic simulations, and show the full image-fit and residuals for each TA image shown in the main text, along with the system of kinetic equations used to fit them.

The global fit algorithm used here was custom written for this study using Igor Pro 8, and deserves some explanation. Fitting a system of ODEs to time resolved data is a common and often laborious task. The main problem is that numerical integration of ODEs tends to be slow, and poorly behaved from the perspective of typical gradient-descent fit algorithms such as Levenberg-Marquardt. If numerical tolerance factors are lowered to accelerate the ODE integration this can generate numerical noise in the output solution, causing the fit algorithm to fail. More seriously there are very often parameter values not too far from the desired solution that will cause the ODE integration to fail. We have found that abandoning gradient descent algorithms in favor of simulated annealing to be a better approach. A simulated annealing algorithm is built into Igor's 'Optimize' operation, a key feature of which is the ability to limit the search range for each parameter so that the user can prevent the algorithm from exploring unphysical, or numerically disastrous, parameter values. While similar constraints can be provided to Igor's CurveFit operation, our experience is that these often cause other problems in fit convergence. Simulated annealing has proven to be more robust, and it is the predominant method used here.

At each iteration of the ODE fitting procedure a solution to your system of ODEs is calculated for a given set of parameters. The solution is a set of species trajectories in time. Each kinetic slice of data is then fit using a linear combination of these (normalized) trajectories, 
which may optionally not include all of them. $\chi^{2}$ for the whole iteration is given by a sum over the variances between these fits and all the kinetic slices under consideration. The result is a set of amplitude values, one for each trajectory on each kinetic slice. When the kinetic slices are taken line by line from a whole image these amplitude values form species-associated spectra.

The overall fitting procedure consists of:

(1) Selecting representative kinetic slices that appear to provide unique dynamical information.

(2) Fitting the system of ordinary differential equations (ODEs) to the slices.

(3) Fitting the ODE solution obtained in this way to the whole image and calculating the residuals and species-associated spectra.

By fitting the system of ODEs to only a handful of representative kinetic slices, a great deal of computational time is saved with little loss of accuracy, and an opportunity arises to select which species contribute to a given slice using the researcher's knowledge of the transient spectra. This is especially useful in the context of ground-state bleach contributions, where the spectrum is easily anticipated.

\section{Specific Equations and Fits}

In every case (TRMC and TA data) excitations are introduced into the sample using a generation function that simulates a gaussian laser pulse, given by:

$$
g(t)=\frac{1}{\sqrt{2 \pi} \sigma} e^{-\left(\frac{t-t_{0}}{\sigma}\right)^{2} / 2}
$$

Where $\sigma$ is the gaussian width of the peak, typically 115 fs for our TA instrument, or $1.7 \mathrm{~ns}$ for TRMC. In the simulation this normalized distribution is multiplied by an excitation density, denoted $N_{0}$, possibly with the addition of a branching ratio $B_{i}$ for cases where ultrafast dynamics 
are inferred to occur inside the laser pulse. For all experiments laser power density was measured at the sample position. Illuminated area in TA was determined by measuring the FWHM of the spot using a set of pinholes. In TRMC the total illuminated sample area is known. In both cases a film thickness of $150 \mathrm{~nm}$ (determined from our previous work) ${ }^{1}$, and the absorptance of the sample at the excitation wavelength (Figure 1(c)) were used to calculate the excitation density in each sample.

We use the following conventions in our kinetic modeling. The symbol $k$ is used to indicate a first-order rate constant, while the symbol $\gamma$ indicates a second-order rate constant. Ground-state bleach (GSB) populations are calculated explicitly, and these are the only species allowed to contribute negative amplitude to fit the TA images and kinetic slices. In addition, our code gives us the freedom to constrain which species contribute to specific kinetic slices. For example, in much of the work below we only allow the GSB and $\mathrm{S}_{1}$ states of PbPc to contribute to the dynamics between 750 and $800 \mathrm{~nm}$. 


\section{Neat Pentacene (TA):}

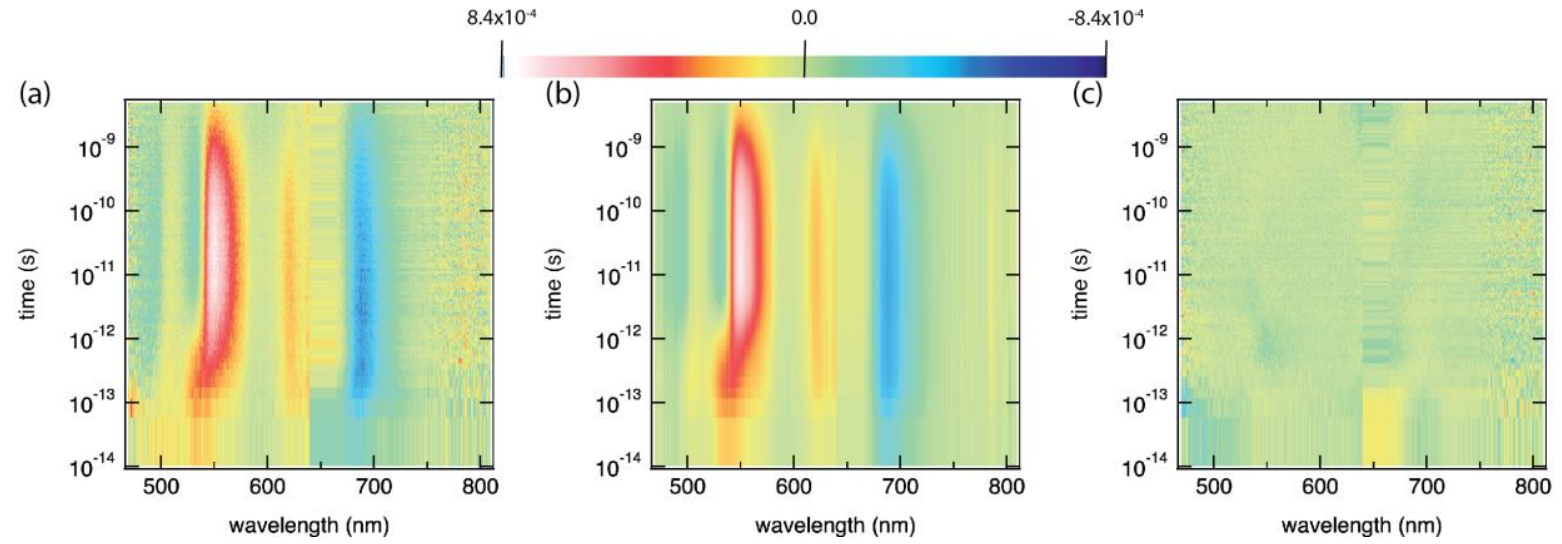

S3.1 (a) TA image for a neat pentacene film. (b) A global fit to the TA data using the kinetic scheme shown below. (c) fit residuals. Fit coefficients: $k_{[T T]-T+T}=5.2 \times 10^{11} \mathrm{~s}^{-1}, \gamma_{T T A}=1.65 \times 10^{-11}$ $\mathrm{cm}^{3} \mathrm{~s}^{-1} .650 \mathrm{~nm}$ excitation at $6 \times 10^{14} \mathrm{~cm}^{-2}$ abs. fluence.

Kinetic scheme used to fit the TA data in figure S3.1

$$
\begin{aligned}
& \frac{d[G]}{d t}=-N_{0} g(t)+k_{[T T] \rightarrow T+T}[T T]+\gamma_{T T A}[T] \\
& \frac{d[T T]}{d t}=N_{0} g(t)-k_{[T T] \rightarrow T+T}[T T] \\
& \frac{d[T]}{d t}=2 k_{[T T] \rightarrow T+T}[T T]-\gamma_{T T A}[T]
\end{aligned}
$$




\subsection{1 $\mathrm{m} \mathrm{PbPc}$ in polystyrene (TA):}

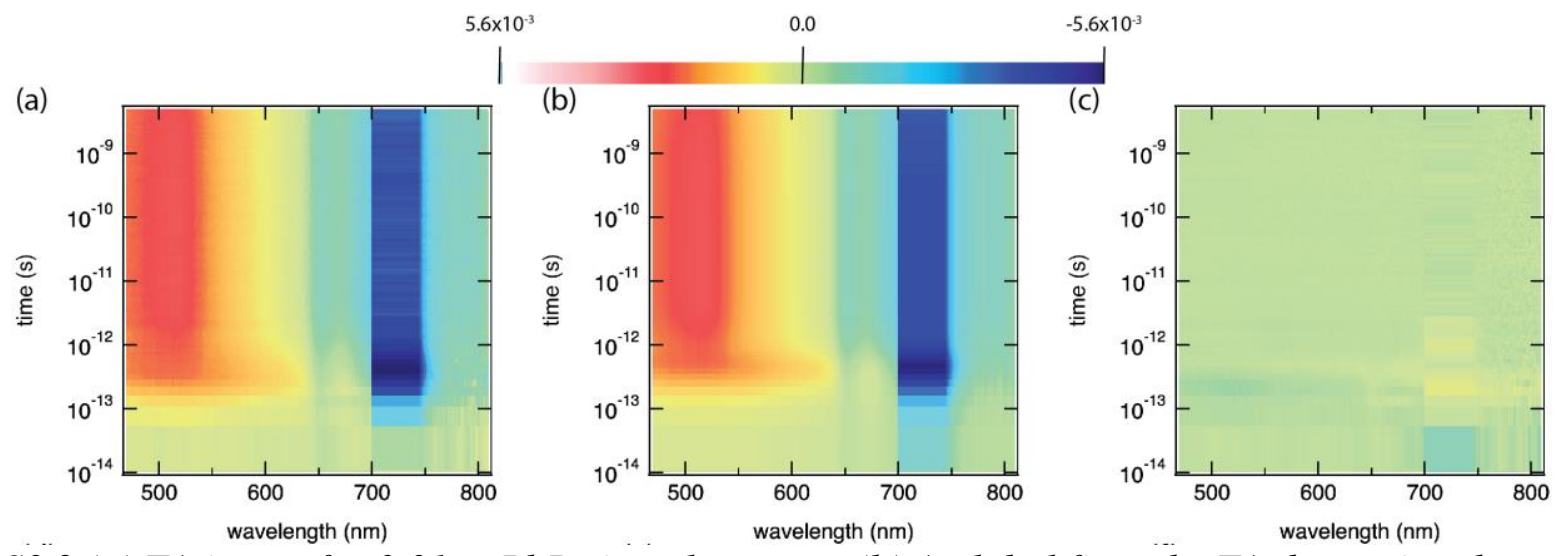

S3.2 (a) TA image for $0.01 \mathrm{mPbPc}$ in polystyrene. (b) A global fit to the TA data using the

kinetic scheme shown below. (c) fit residuals. Fit coefficients: $k_{I S C}=9.3 \times 10^{11} \mathrm{~s}^{-1}, \gamma_{S S A}=2.7 \times 10^{-7}$ $\mathrm{cm}^{3} \mathrm{~s}^{-1,} \mathrm{k}_{r}=1 \times 10^{7} \mathrm{~s}^{-1} .725 \mathrm{~nm}$ excitation at $3.2 \times 10^{14} \mathrm{~cm}^{-2}$ abs. fluence.

Kinetic scheme used to fit the TA data in Figure S3.2

$$
\begin{aligned}
& \frac{d[G]}{d t}=-N_{0} g(t)+\gamma_{S S A}[S]^{2}+k_{r}[T] \\
& \frac{d[S]}{d t}=N_{0} g(t)-k_{I S C}[S]-\gamma_{S S A}[S]^{2} \\
& \frac{d[T]}{d t}=k_{I S C}[S]-k_{r}[T]
\end{aligned}
$$




\subsection{5 m PbPc in Pentacene (TA):}

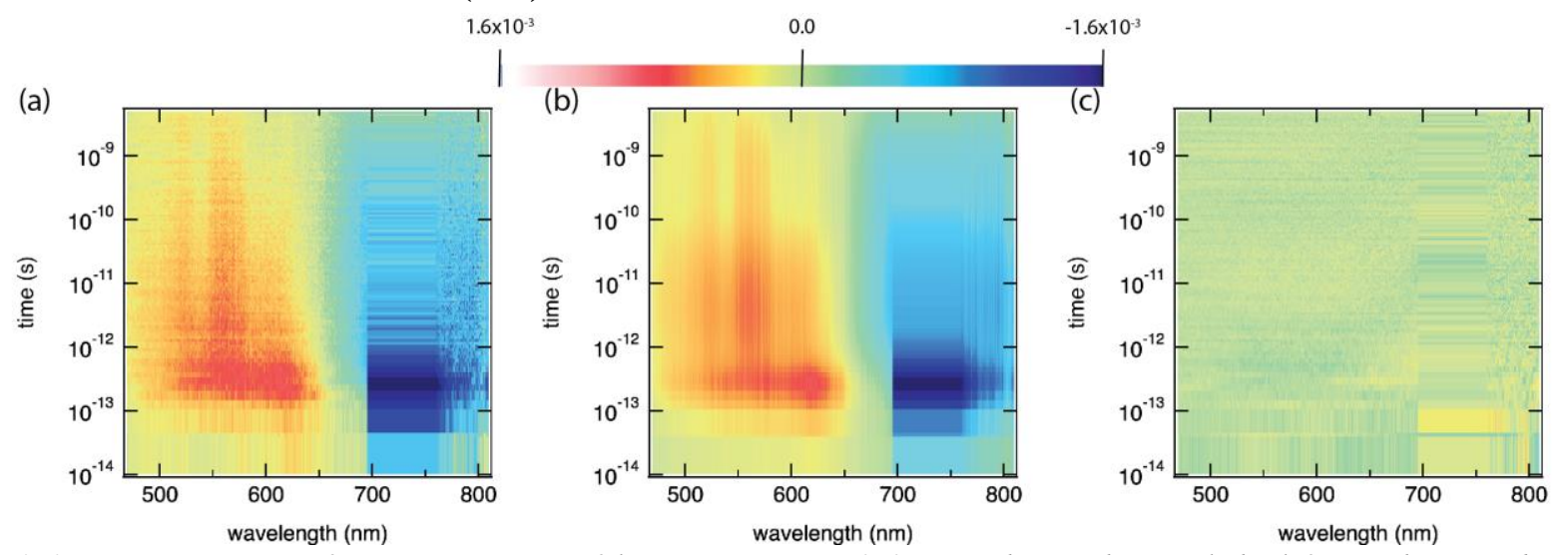

S3.3: (a) TA image for a pentacene film containing $0.05 \mathrm{~m} \mathrm{PbPc}$. (b) A global fit to the TA data using the kinetic scheme shown below. (c) fit residuals. Fit coefficients: $k_{T E T[a]}=2.0 \times 10^{10} \mathrm{~s}^{-1}$, $k_{T E T[b]}=1.3 \times 10^{8} \mathrm{~s}^{-1}, B_{T[a / b]}=0.35 .725 \mathrm{~nm}$ excitation at $3.2 \times 10^{14} \mathrm{~cm}^{-2}$ abs. fluence.

Kinetic scheme used to fit the TA data in Figure S3.3

$$
\begin{aligned}
& \frac{d\left[G_{P c}\right]}{d t}=-N_{0} g(t)+\gamma_{S S A}[S]^{2}+k_{r[P c]}\left(\left[T_{P c[a]}\right]+\left[T_{P c[b]}\right]\right)+k_{T E T[a]}\left[T_{P c[a]}\right]+k_{T E T[b]}\left[T_{P c[b]}\right] \\
& \frac{d[S]}{d t}=N_{0} g(t)-k_{I S C}[S]-\gamma_{S S A}[S]^{2} \\
& \frac{d\left[T_{P c[a]}\right]}{d t}=B_{T[a / b]} k_{I S C}[S]-k_{r[p c]}\left[T_{P c[a]}\right]-k_{T E T[a]}\left[T_{P c[a]}\right] \\
& \frac{d\left[T_{P c[b]}\right]}{d t}=\left(1-B_{T[a / b]}\right) k_{I S C}[S]-k_{r[p c]}\left[T_{P c[b]}\right]-k_{T E T[b]}\left[T_{P c[b]}\right] \\
& \frac{d\left[G_{P t c}\right]}{d t}=-k_{T E T[a]}\left[T_{P c[a]}\right]-k_{T E T[b]}\left[T_{P c[b]}\right]+\gamma_{T T A}\left[T_{P t c}\right]^{2} \\
& \frac{d\left[T_{P t c}\right]}{d t}=k_{T E T[a]}\left[T_{P c[a]}\right]+k_{T E T[b]}\left[T_{P c[b]}\right]-\gamma_{T T A}\left[T_{P t c}\right]^{2}
\end{aligned}
$$




\section{$0.05 \mathrm{~m} \mathrm{PbPc}$ and $0.05 \mathrm{~m}$ PDI in polystyrene (TA):}
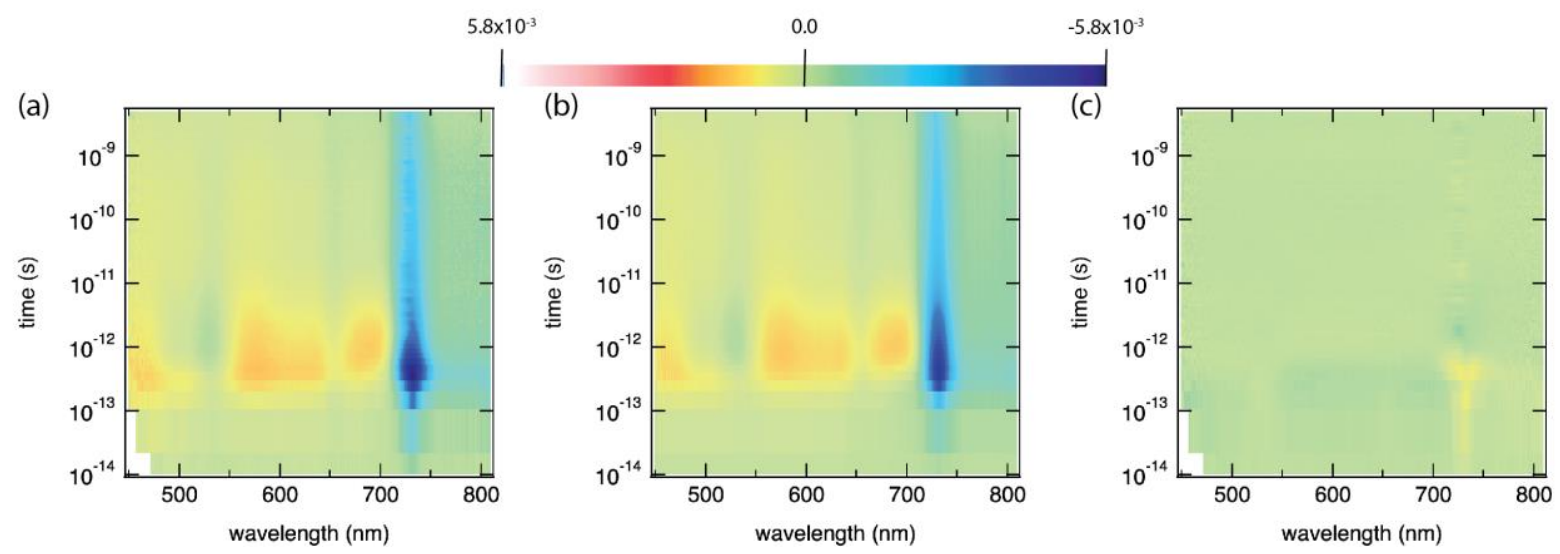

S3.4: (a) TA image for a polystyrene film containing $0.05 \mathrm{~m}$ PbPc and $0.05 \mathrm{~m}$ PDI. (b) A global fit to the TA data using the kinetic scheme shown below. (c) fit residuals. Fit coefficients: $\gamma_{C R}=$ $2.6 \times 10^{-7} \mathrm{~cm}^{3} \mathrm{~s}^{-1}, \gamma_{T C A}=9 \times 10^{-9} \mathrm{~cm}^{3} \mathrm{~s}^{-1}, B_{C T}=0.31, k_{T E T}=5 \times 10^{6} \mathrm{~s}^{-1} .725 \mathrm{~nm}$ excitation at $2.1 \times 10^{14}$ $\mathrm{cm}^{-2}$ abs. fluence.

Kinetic scheme used to fit the TA data in Figure S3.4

$$
\begin{aligned}
& \frac{d\left[G_{P c}\right]}{d t}=-N_{0} g(t)+\gamma_{S S A}[S]^{2}+k_{r}\left[T_{P c}\right]+\gamma_{T C A}\left[T_{P c}\right]\left[P c^{+} P D I^{-}\right]+\gamma_{C R}\left[P c^{+} P D I^{-}\right]^{2}+k_{T E T}\left[T_{P c}\right] \\
& \frac{d[S]}{d t}=\left(1-B_{C T}\right) N_{0} g(t)-k_{I S C}[S]-\gamma_{S S A}[S]^{2} \\
& \frac{d\left[T_{P c}\right]}{d t}=k_{I S C}[S]-k_{r}\left[T_{P c}\right]-\gamma_{T C A}\left[T_{P c}\right]\left[P c^{+} P D I^{-}\right]-k_{T E T}\left[T_{P c}\right] \\
& \frac{d\left[P c^{+} P D I^{-}\right]}{d t}=B_{C T} N_{0} g(t)-\gamma_{C R}\left[P c^{+} P D I^{-}\right]^{2} \\
& \frac{d\left[G_{P D I}\right]}{d t}=-B_{C T} N_{0} g(t)-k_{T E T}\left[T_{P c}\right]+\gamma_{C R}\left[P c^{+} P D I^{-}\right]^{2}
\end{aligned}
$$




\subsection{5 $\mathrm{m}$ PbPc and 0.05 m PDI in Pentacene (TA):}

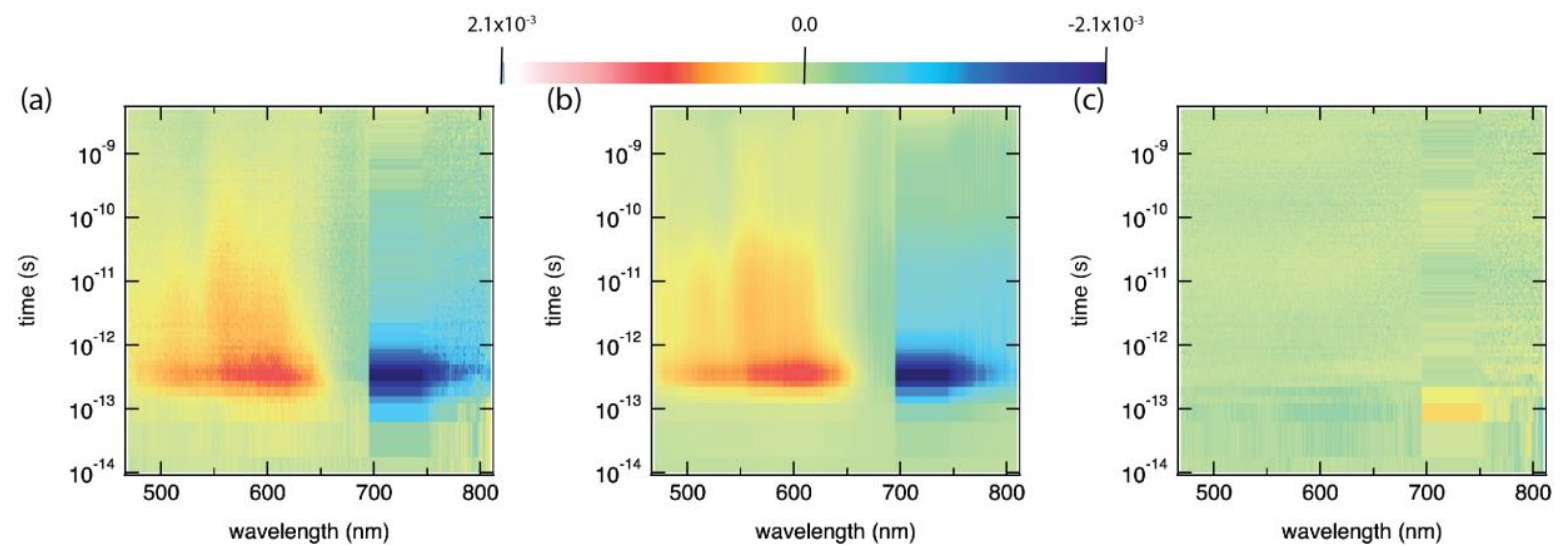

S3.5: (a) TA image for a pentacene film containing $0.05 \mathrm{~m} \mathrm{PbPc}$ and $0.05 \mathrm{~m}$ PDI. (b) A global fit to the TA data using the kinetic scheme shown below. (c) fit residuals. Fit coefficients: $B_{C T}=$ $2 \times 10^{-5} .725 \mathrm{~nm}$ excitation at $3.2 \times 10^{14} \mathrm{~cm}^{-2}$ abs. fluence.

Kinetic scheme used to fit the TA data in Figure S3.5

$$
\begin{aligned}
& \frac{d\left[G_{P c}\right]}{d t}=-\left(1-B_{C T}\right) N_{0} g(t)+\gamma_{S S A}[S]^{2}+k_{r[P c]}\left(\left[T_{P c[a]}\right]+\left[T_{P c[b]}\right]\right)+k_{T E T[a]}\left[T_{P c[a]}\right]+k_{T E T[b]}\left[T_{P c[b]}\right] \\
& +\gamma_{T C A}\left(\left[T_{P c[a]}\right]+\left[T_{P c[b]}\right]\right)\left[\operatorname{Ptc}^{+} P D I^{-}\right] \\
& \frac{d[S]}{d t}=\left(1-B_{C T}\right) N_{0} g(t)-k_{I S C}[S]-\gamma_{S S A}[S]^{2} \\
& \frac{d\left[T_{P c[a]}\right]}{d t}=B_{T[a / b]} k_{I S C}[S]-k_{r}\left[T_{P c[a]}\right]-k_{T E T[a]}\left[T_{P c[a]}\right]-\gamma_{T C A}\left[T_{P c[a]}\right]\left[P t c^{+} P D I^{-}\right] \\
& \frac{d\left[T_{P c[b]}\right]}{d t}=\left(1-B_{T[a / b]}\right) k_{I S C}[S]-k_{r}\left[T_{P c[b]}\right]-k_{T E T[b]}\left[T_{P c[b]}\right]-\gamma_{T C A}\left[T_{P c[b]}\right]\left[P t c^{+} P D I^{-}\right] \\
& \frac{d\left[G_{P t c}\right]}{d t}=-B_{C T} N_{0} g(t)-k_{T E T[a]}\left[T_{P c[a]}\right]-k_{T E T[b]}\left[T_{P c[b]}\right]+\gamma_{T T A}\left[T_{P t c}\right]^{2}+\gamma_{C R}\left[P t c^{+} P D I^{-}\right]^{2} \\
& +\boldsymbol{k}_{C R}\left[\mathrm{Ptc}^{+} \mathrm{PDI}^{-}\right] \\
& \frac{d\left[T_{P t c}\right]}{d t}=k_{T E T[a]}\left[T_{P c[a]}\right]+k_{T E T[b]}\left[T_{P c[b]}\right]-\gamma_{T T A}\left[T_{P t c}\right]^{2}-k_{C T}\left[T_{P t c}\right]-\gamma_{T C A}\left[P t c^{+} P D I^{-}\right]\left[T_{P t c}\right] \\
& \frac{d\left[\mathrm{Ptc}^{+} \mathrm{PDI}^{-}\right]}{d t}=B_{C T} N_{0} g(t)+k_{C T}\left[T_{P t c}\right]-\gamma_{C R}\left[\mathrm{Ptc}^{+} \mathrm{PDI}^{-}\right]^{2}
\end{aligned}
$$




\subsection{5 m PbPc and $0.05 \mathrm{~m}$ PDI in pentacene (TRMC):}

Kinetic Scheme used to fit the TRMC data in main text Figure 8:

$$
\begin{aligned}
& \frac{d\left[G_{P c}\right]}{d t}=-\left(1-B_{C T}\right)\left(1-B_{P t c_{-} e x}\right) N_{0} g(t)+\gamma_{S S A}[S]^{2}+k_{r[P c]}\left(\left[T_{P c[a]}\right]+\left[T_{P c[b]}\right]\right)+k_{T E T[a]}\left[T_{P c[a]}\right] \\
& +\boldsymbol{k}_{T E T[b]}\left[\boldsymbol{T}_{P b P c[b]}\right]+\gamma_{T C A}\left(\left[\boldsymbol{T}_{P c[a]}\right]+\left[\boldsymbol{T}_{P c[b]}\right]\right)\left[\operatorname{Ptc}^{+} \boldsymbol{P D I}^{-}\right] \\
& \frac{d[S]}{d t}=\left(1-B_{C T}\right)\left(1-B_{P t c_{-} e x}\right) N_{0} g(t)-k_{I S C}[S]-\gamma_{S S A}[S]^{2} \\
& \frac{d\left[T_{P c[a]}\right]}{d t}=B_{T[a / b]} k_{I S C}[S]-k_{r}\left[T_{P c[a]}\right]-k_{T E T[a]}\left[T_{P c[a]}\right]-\gamma_{T C A}\left[T_{P c[a]}\right]\left[P t c^{+} P D I^{-}\right] \\
& \frac{d\left[T_{P c[b]}\right]}{d t}=\left(1-B_{T[a / b]}\right) k_{I S C}[S]-k_{r}\left[T_{P c[b]}\right]-k_{T E T[b]}\left[T_{P c[b]}\right]-\gamma_{T C A}\left[T_{P c[b]}\right]\left[P t c^{+} P D I^{-}\right] \\
& \frac{d\left[G_{P t c}\right]}{d t}=-\left(B_{C T}\left(1-B_{P t c_{e x}}\right)+B_{P t c_{e x}}\right) N_{0} g(t)-k_{T E T[a]}\left[T_{P c[a]}\right]-k_{T E T[b]}\left[T_{P c[b]}\right]+\gamma_{T T A}\left[T_{P t c}\right]^{2} \\
& +\gamma_{C R}\left[\operatorname{Ptc}^{+} \boldsymbol{P D I ^ { - }}\right]^{2}+\boldsymbol{k}_{C R}\left[\boldsymbol{P t c}^{+} \boldsymbol{P D I ^ { - }}\right] \\
& \frac{d\left[T_{P t c}\right]}{d t}=2 B_{P t c_{e x}} N_{0} g(t)+k_{T E T[a]}\left[T_{P c[a]}\right]+k_{T E T[b]}\left[T_{P c[b]}\right]-\gamma_{T T A}\left[T_{P t c}\right]^{2}-k_{C T}\left[T_{P t c}\right] \\
& -\gamma_{T C A}\left[P_{t c}^{+} P^{-}\right]\left[T_{P t c}\right] \\
& \frac{d\left[P_{t c}^{+} P D I^{-}\right]}{d t}=B_{C T}\left(1-B_{P t c_{-} e x}\right) N_{0} g(t)+k_{C T}\left[T_{P t c}\right]-\gamma_{C R}\left[P t c^{+} P D I^{-}\right]^{2}-k_{C R}\left[P t c^{+} P D I^{-}\right]
\end{aligned}
$$




\section{Kinetic Monte Carlo Modeling of Diffusion-Limited Effective Charge Transfer Rate}

\section{Constants:}
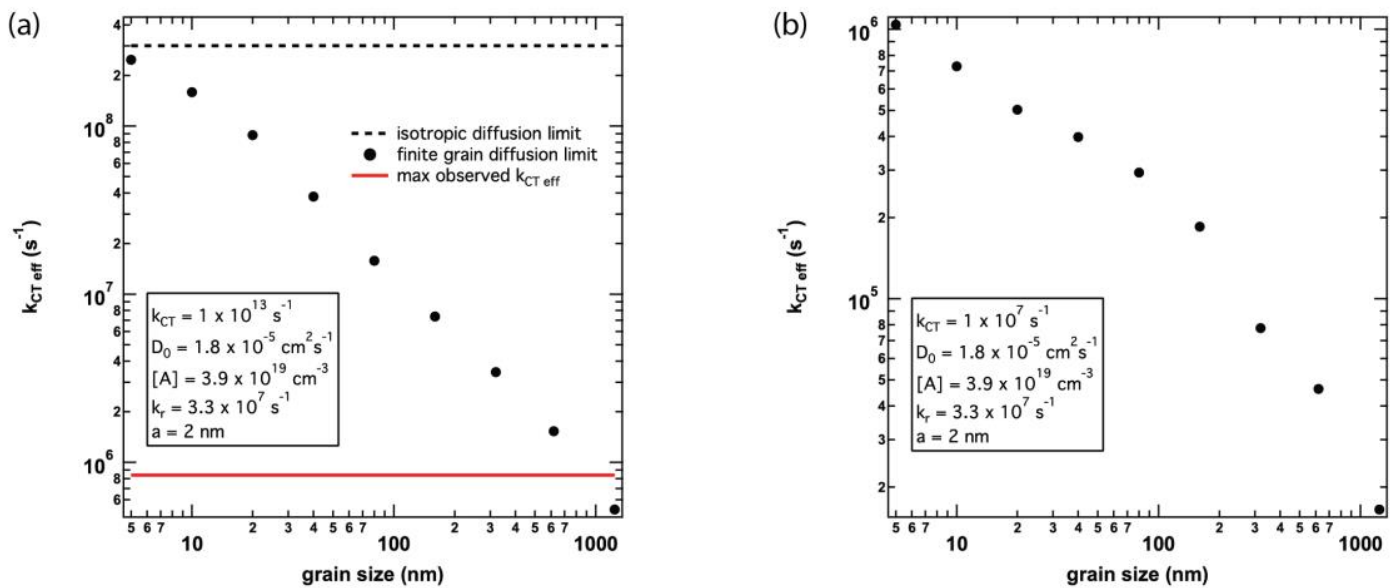

S4: (a) kMC calculations showing the diffusion-limit for the effective charge transfer rate constant. Two situations are simulated: the isotropic case (dashed black line) and the phasesegregated case where finite spherical grains of pure pentacene exclude the acceptor molecules, which are randomly distributed on their surface (black circles). The red line shows the maximum effective charge transfer rate constant we observe in our experiments. (b) The same simulation, with a small finite fundamental charge-transfer rate-constant $\left(10^{7} \mathrm{~s}^{-1}\right)$ showing how the effective charge transfer rate constant depends on grain size, even when charge transfer events are not ultrafast. In each case $k_{C T}$ is the fundamental charge-transfer rate constant, $D_{0}$ is the exciton diffusion coefficient, [A] is the acceptor concentration, $k_{r}$ is the effective triplet decay rate constant, and $a$ is the site-size in the simulation.

\section{References:}

(1) Pace, N. A.; Korovina, N. V.; Clikeman, T. T.; Holliday, S.; Granger, D. B.; Carroll, G. M.; Nanayakkara, S. U.; Anthony, J. E.; McCulloch, I.; Strauss, S. H.; et al. Slow Charge Transfer from Pentacene Triplet States at the Marcus Optimum. Nature Chemistry 2019. 